\title{
BOND GRAPHS MODELLING OF ELECTROMAGNETIC ACTUATORS
}

\author{
Constantin Niţu1, Bogdan Grămescu², Mihai Avram³ \\ 1,2,3 University POLITEHNICA of Bucharest \\ 313, Splaiul Independenţei, 060042-Bucharest, Romania \\ constantin.nitu@upb.ro
}

\begin{abstract}
The paper presents the model of an electromagnetic actuator, developed in $20 \mathrm{sim}$ environment, by use of Bond Graph method. The software facilities are exploited by using both signal block diagram and the power bond graph elements within the same model. The models of the flux raising stage, when the armature doesn't move and the dynamic period, until the armature ends its stroke are presented. These ones take into account only the saturation of the core magnetization curve, but not its nonlinearity. The data used for simulation are from an experimental tested device, so, both calculated and measured results can be compared.
\end{abstract}

Keywords: Bond Graphs, Mechatronics, Electromagnetic Actuator, Dynamic Behaviour, 20 SIM.

\section{Introduction}

Many industrial applications make use of binary state actuators, with limited displacement, which are electrically driven. Hydraulic/pneumatic distributors, intake/exhaust valves for camless variable distribution of the spark ignition engines, electric power switching devices or mobile robots are common examples [1], [2], [3]. Their main feature is quick switching between two stable positions, one stroke being obtained by electromagnetic forces, while the return is accomplished by help of springs. Sometimes, they have to keep one of the two stable positions for a shorter or longer period, according to the operation cycle of the system to which they belong.

Some applications, like electromagnetic actuation of the intake and exhaust valves of the automobile engines, meant to contribute to the fuel economy, power increasing and pollution diminishing, enable computer control of the valves motion as concerns stroke, duration of the movement and frequency, following programmable laws as the essential difference from the actual mechanisms which have fixed and previously determined laws [4].

\section{Actuator Model}

An electromagnetic actuator is, in fact, a mechatronic system, which operates in transient regime.
It consists of the driving circuit, the electromagnet and the driven mechanism. Its regular use is for the applications which require small strokes, constant amplitude and high frequency. Some typical examples are related to the hydraulic/pneumatic valves and electromechanical switching devices.

During the last years, a special interest for this type of actuating was shown in the automotive industry for actuating the intake/exhaust valves of the thermal engines. When modeling the operation of such actuators, one should take mainly into consideration the position, velocity and acceleration of the moving parts, in different stages of an operation cycle, as well as the current and generated force evolution, power consumption, duration and settling time.

Mechatronic approach is obviously because the equations describing the electric circuit, magnetic behavior and the mechanism dynamics have to be solved simultaneously.

The simplest driver for an inductive load, with a constant voltage applied to the coil, is shown in figure 1. It is based on the switching operation of the transistor, which is open for the period $\tau$, when the electric circuit equation is:

$$
u=\frac{d \lambda}{d t}+R i
$$

where: $\lambda=N \varphi$ - magnetic flux linkage in the coil; $\mathrm{N}$ number of coil turns; $i$ - current; $R$ - electric resistance of the coil; $u=V_{C}-V_{C E}$. 

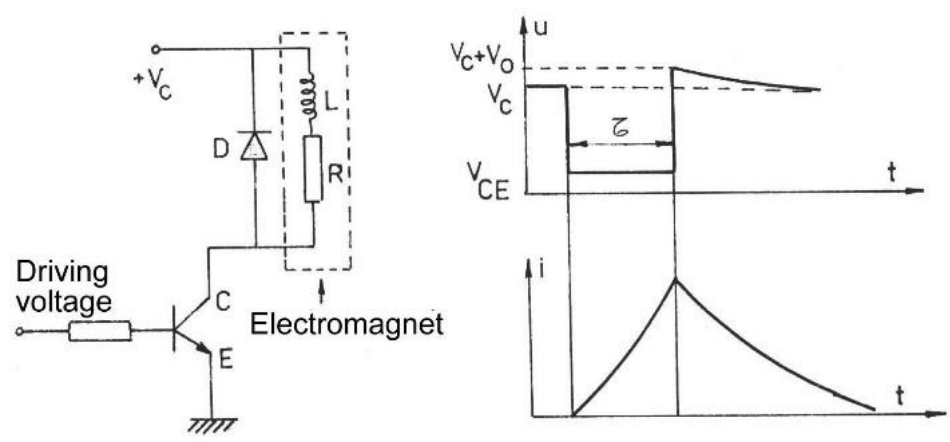

Fig. 1. Driving circuit with voltage step

After canceling the driving voltage, $u$, current variation and magnetic flux one could be obtained from the equation:

$$
\frac{d \lambda}{d t}+R i+V_{0}=0
$$

where $V_{0}$ is the diode theshold voltage. This equation characterizes the slow current decrease in the loop R, L, D, after the conduction of the CE junction is interrupted. Because during this period there is also a power transfer, the driving circuit is an efficient and cost-effective one, but it has low actuating frequency. Better results for current supressing could be obtained by use of a zener diode in series with the regular one. Such circuit is also suited, in principle, for a chopped driving when rectangular pulses are applied to the coil. So, the equations (1) and (2) define the electric evolution of the system, if they are used in successive periods of time [5].

The electromagnetic actuator is particularly interesting when modeling the magnetic circuit. Usually, the physical model is represented in terms of reluctances or permeances of the magnetic core. This is due to the regular analogy, which makes the well-known correspondence magnetic flux-current and magnetomotive force-voltage. The result is that the reluctance seems to have a dissipative character, while it is known the device is storing the energy. Another approach is proposed in [6], by taking as magnetic through variable the derivative of the flux linkage, $\lambda$. The magnetomotive force, which sets up the magnetic flux $\varphi$ in the yoke is related to the current through the coil by $M=N . i$. In the same time, for a part of the magnetic core or air gap, the magnetomotive force drop is:

$$
M=\frac{\varphi}{P}=\frac{1}{P} \int \dot{\varphi} d t
$$

where $P$ is the permeance (inverse of the reluctance). This equation shows an analogy between permeance and spring compliance or electric capacity, respectively.
When evaluating reluctances or permeances, Rotters's method of volumes is used and it results generally as $P=\mu A / l$, where: $\mu$ - magnetic core permeability; $A_{c}$ - cross section area; $l$ - length og the magnetic path (figure 2). All the parameters refer to the core section or air gap.



Fig. 2. Volume method for reluctance calculation

The magnetic path can be divided into parts crossed by the same magnetic flux, in order to calculate the permeances of the actuator core, moving armature or air gap. But the time variation of the magnetic flux is generating eddy currents inside the magnetic core (figure 3), which also create a magnetic effect trying to oppose to the magnetic flux increase.

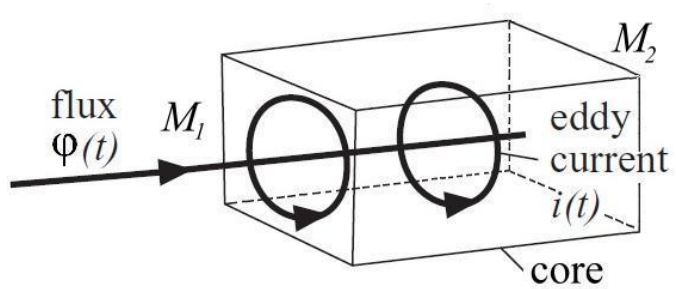

Fig. 3. Eddy current generation into magnetic core

A model which take into consideration the influence of eddy currents upon the magnetic flux variation and losses was developed by Schweer [7] and simply express the magnetomotive force between 2 points of the magnetic path (figure 3 ) as:

$$
M_{1}-M_{2}=\frac{\varphi}{P}+G_{e d d y} \cdot \dot{\varphi}=\frac{1}{P} \int \dot{\varphi} d t+G_{e d d y} \cdot \dot{\varphi}
$$


where $G_{e d d y}$ is the electric conductance of the eddy current path into the magnetic core.

The magnetic permeability of the actuator magnetic path, used for the calculation of permeances, depends on the slope $\mu$ of the magnetization curve, $B-H$. For a free space this one is constant $\left(\mu_{0}\right)$, while for a ferromagnetic core can be considered constant only within a limited area, around the origin (figure 4).


Fig. 4. Magnetization curves for free space and magnetic materials (real and linearized)
For simplifying the model, the $B$ - $H$ curve for the core material is linearly modelled with saturation (figure 4 ), according to the equation:

$$
B=\left\{\begin{array}{l}
B_{\text {sat }} \text { for } H \geq B_{\text {sat }} / \mu \\
\mu H \text { for }|H|<B_{\text {sat }} / \mu \\
-B_{\text {sat }} \text { for } H \leq-B_{\text {sat }} / \mu
\end{array}\right.
$$

This means the magnetic flux will have a saturation value, $\varphi_{\text {sat }}=B_{\text {sat }} A_{c}$, which will be used for electromagnetic force calculation, regardless the electric current increase. This phenomenon is supposed to appear when the air gap is very small and the current in the coil is high.

\section{Bond Graph Modelling}

Bond Graphs is a method to represent the power flows in mechatronic systems, which handles generalized power-variables: effort $\boldsymbol{e}$ and flow $\boldsymbol{f}$, as well as momentum $\boldsymbol{p}$ (integral of effort) and displacement $\boldsymbol{q}$ (integral of flow).

The power flow between different subsystems is represented by a 'bond' looking like a half arrow.

An example of a word bond graph of the electromagnetic actuator is shown in figure 5 .

\section{Controlled electrical power

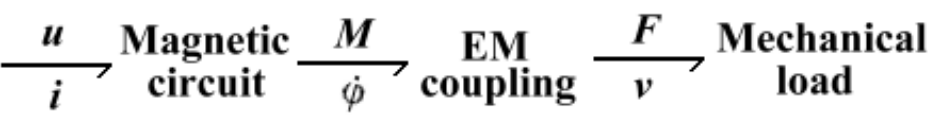

Fig. 5. Word bond graph of the electromagnetic actuator

In a lumped parameter model, each subsystem consists of basic elements, which can be classified in passive and active elements. There are 3 types of passive elements, defined from the electromechanical analogy, when taking the force (torque) in the mechanical domain as effort variable and velocity (angular velocity) for flow variable.

Active elements are sources of effort $S e$ and sources of flow $S f$. If their output is controlled by a signal, they are called "modulated" (MSe and MSf, respectively). Elements can be connected with 0junctions (common effort, as in a node of an electric circuit), 1-junctions (common flow, as in a series electric circuit), transformers (TF) and gyrators (GY). These junctions are considered as being power conservative. A 0 -junction and a 1-junction are shown in figure 6.
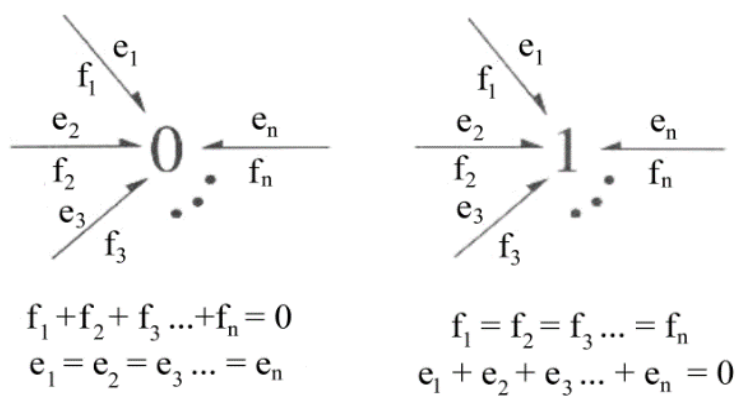

$\mathrm{f}_{1}+\mathrm{f}_{2}+\mathrm{f}_{3} \ldots+\mathrm{f}_{\mathrm{n}}=0$

$\mathrm{e}_{1}=\mathrm{e}_{2}=\mathrm{e}_{3} \ldots=\mathrm{e}_{\mathrm{n}}$

$\mathrm{e}_{1}+\mathrm{e}_{2}+\mathrm{e}_{3} \ldots+\mathrm{e}_{\mathrm{n}}=0$

Fig. 6. 0-junction and 1-junction rules

The geometry of the modelled electromagnetic actuator is presented in figure 7 , and it is used to evaluate the permeances and the resistance of the eddy currents path within the magnetic core. 
The translational motion of the armature is suited for hydraulic/pneumatic valves actuation and a spring compliance and damping effect were added in order to model the behaviour of an elastic element.

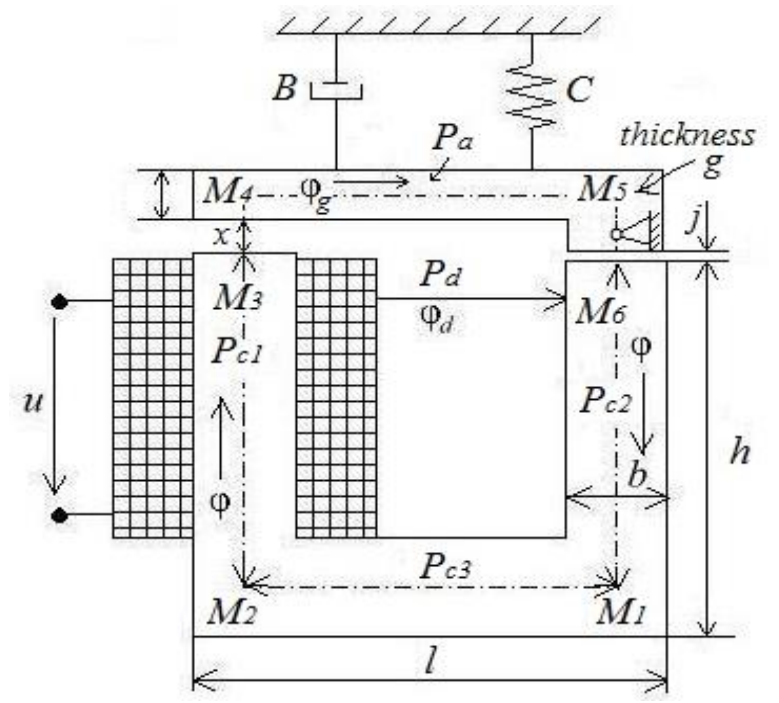

Fig. 7. Electromagnetic actuator

The core reluctances, $R_{c 1}, R_{c 2}, R_{c 3}$ and the moving armature one, $R_{a}$, are calculated according to the figures 2 and 7 and inverted to the corresponding permeances:

$P_{c 1}=P_{c 2}=\frac{\mu b g}{h-b / 2} ; P_{c 3}=\frac{\mu b g}{l-b} ; P_{a}=\frac{\mu a g}{l-b}$

According to the equation (3), the bond graph representation of these permeances is the general $C$ storage element, while the losses caused by eddy currents are taken into consideration by means of dissipative $R$ elements (figure 8).

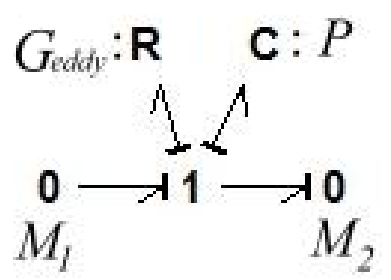

Fig. 8. Bond Graph representation of equation (3)

A core electrical conductance is evaluated, by assuming an average eddy current path, for each core part. It results:

$$
\begin{aligned}
& G_{e d d y 1}=G_{e d d y 2}=\frac{0.5 b(h-0.5 b) \sigma}{b+g} ; \\
& G_{e d d y 3}=\frac{0.5 b(l-b) \sigma}{b+g} ; \\
& G_{e d d y a}=\frac{0.5 a(l-b) \sigma}{a+g}
\end{aligned}
$$

where $\sigma$ is the electric conductivity of the magnetic core.
Even there are several paths for the magnetic flux dispersion and fringes, regularly they are ignored, excepting the dispersion between the yoke branches (figure 7). The dispersion permeance is calculated with:

$$
P_{d}=\frac{\mu_{0}(h-b) g}{l-2 b}
$$

Another assumption is that the two air gaps and the armature are travelled by the same magnetic flux, $\varphi_{g}$. One air gap permeance, $P_{g 1}$, is variable, depending on $x$ (figure 7) and contributes to the armature displacement, while the second one, $P_{g 2}$, is related to the necessary clearance of the armature joint:

$$
P_{g 1}=\frac{\mu_{0} b g}{x} ; P_{g 2}=\frac{\mu_{0} b g}{j}
$$

When the actuator model is run in the simulation software, several functional stages should be analyzed. These ones are shown in figure 9, where $y$ $=d-x$, is the armature displacement and $d$, initial air gap.

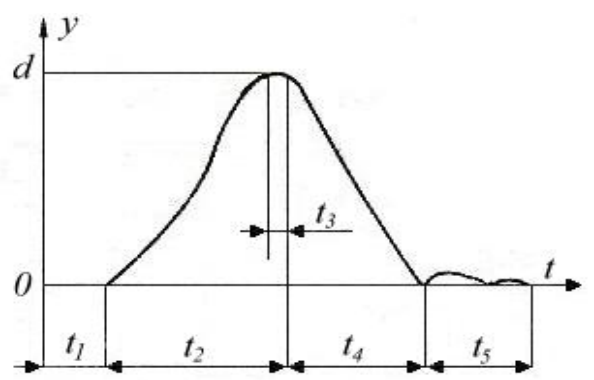

Fig. 9. Armature displacement diagram

The period $t_{1}$ is the necessary duration for magnetic flux raise at the value which can develop an actuation force equal to the resistive one. During this period, there is no displacement of the armature. The period $t_{2}$ is the duration of the armature motion, but it could have different electric equations, depending on the control voltage value. After the stroke is ended, there is a small period for reversing the movement direction, $t_{3}$, which depends on the actuator task. It can be kept only if the actuator coil is energized and it was included in $t_{2}$, as a part of the electromagnetic actuation. The return stroke is achieved in the period $t_{4}$, the movement being the result of spring action. In most cases there is a settling time, $t_{5}$, when the mobile armature could have an oscillation, after collision with its stop part.

For determining the magnetic flux raise in figure 9 ( $t_{1}$ period) and the armature displacement ( $t_{2}$ period), two bond graph models are used: with constant air gap and no armature motion and with variable air gap and armature motion against spring and damper (figure 7). 
The model of the magnetic flux raise consists only of the electric and magnetic circuit/equations and it is presented in figure 10. This is valid for permeance $P_{g 1}$ at maximum air gap $(x=d)$.

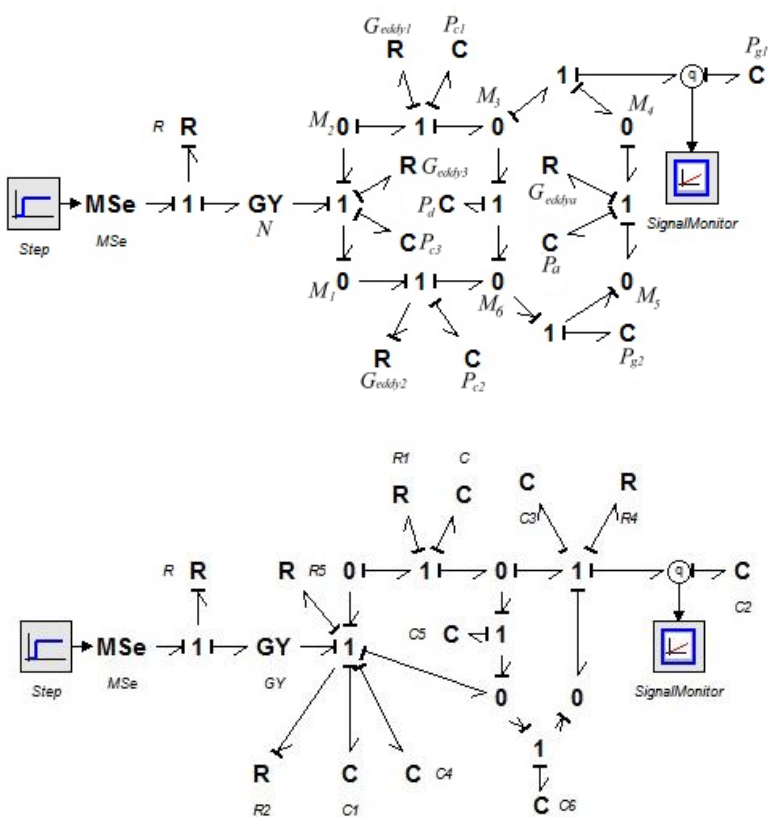

Fig. 10. Bond Graph model of flux raise period before and after simplifying

For the $2^{\text {nd }}$ period, when there is a motion of the armature, a nonlinear $C$ - field element has to be introduced, because the magnetic flux and magnetic force both influence and are depending on the air gap value [6]. The $C$-field is described by the equations which relate the magnetic flux as a generalized momentum, with magnetomotive force and the mechanical displacement as well as with the magnetic force, respectively:

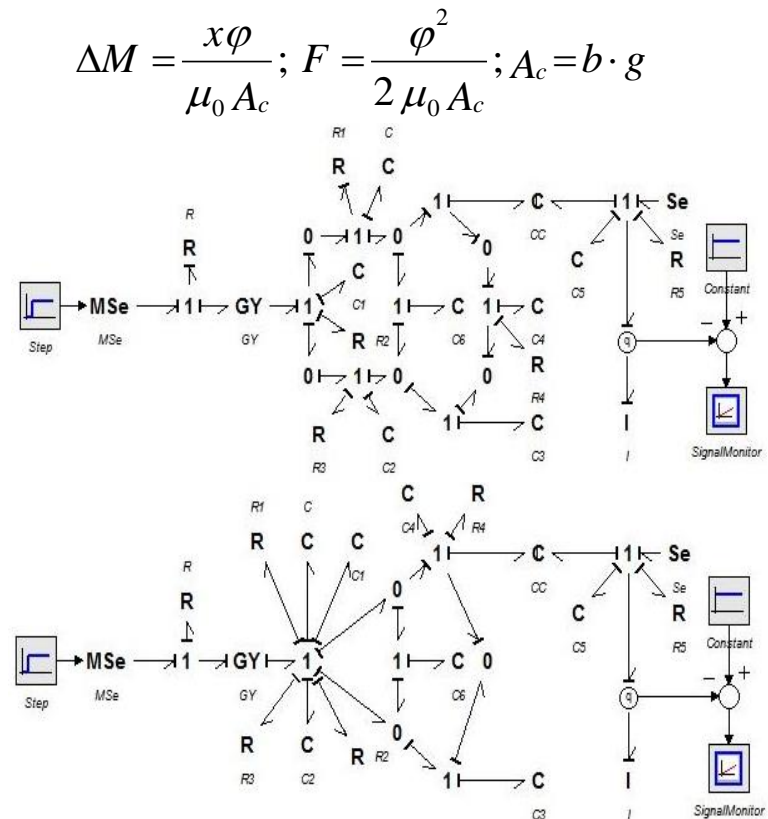

Fig. 11. Bond Graph model of armature motion period before and after simplifying
Both models are implemented in $20 \mathrm{sim}$ software, which can handle Bond Graphs, iconic and signal block diagrams, separately and combined. In the latest version (4.6), it is possible to have a single model for both operating periods ( $t_{1}$ and $\left.t_{2}\right)$, derived from figure 11, if a Switching One Junction (X1) instead of regular 1-junction, is used to connect the $C$-field and the $\mathrm{C}$ storage element $\left(P_{g 1}\right.$ for $\left.x=d\right)$.

Simulation was performed for the electromagnetic actuator for which an improved analytical model was derived [5], having the following parameters: dimensions $l=h=6.3 \times 10^{-3} \mathrm{~m}$, $b=a=2.1 \times 10^{-3} \mathrm{~m}, g=4.2 \times 10^{-3} \mathrm{~m}$; initial air gaps $d=0.35 \times 10^{-3} \mathrm{~m} ; j=0.1 \times 10^{-3} \mathrm{~m}$; iron core with $\mu=875 \times$ $10^{-6} \mathrm{H} / \mathrm{m}, \sigma=9,6 \times 10^{6} \mathrm{~S} / \mathrm{m}$ and $B_{\text {sat }}=1.9 \mathrm{~T}$; magnetic permeability of free space, $\mu_{0}=4 \pi \times 10^{37} \mathrm{H} / \mathrm{m}$; number of coil turns, $N=370$; energizing voltage, $u=40 \mathrm{~V}$; mass of moving armature, $m=0.044 \times 10^{-3} \mathrm{~kg}$; spring compliance, $C=0.11 \mathrm{~m} / \mathrm{N}$; resistant force on the moving armature, at rest position, $F_{r e s}=332 \times 10^{-3} \mathrm{~N}$. By use of equations (1)...(9) with these data, the model parameters were determined.

A simulation result for the magnetic flux raise, by help of the model in figure 10 , is presented in figure 12. The first section of the curve serves for determining the duration $t_{1}$, at the corresponding value of the magnetic flux, calculated with:

$$
\phi_{0}=\sqrt{2 \mu_{0} A_{c} F_{r e s}}
$$
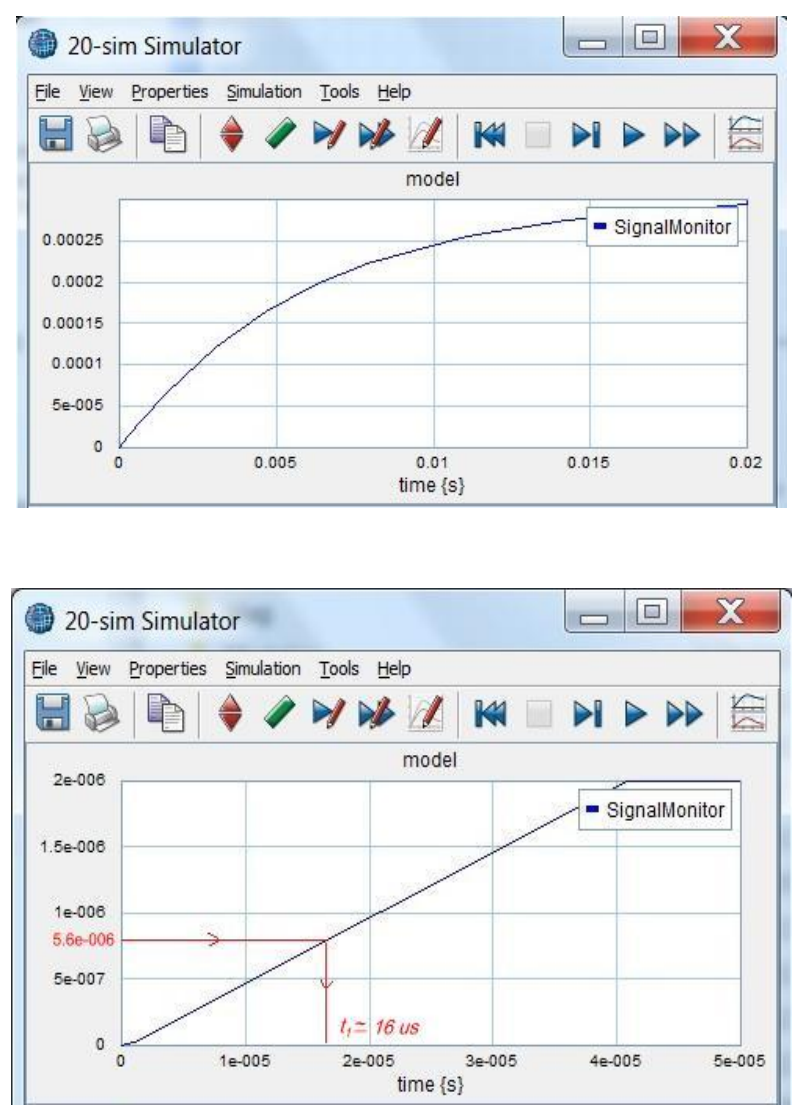

Fig. 12. Magnetic flux raise at no movement 
For the dynamic behaviour of the electromagnetic actuator, the model in figure 11 is run and the period $t_{2}$ is determined. As the air gap length, denoted by $x$, is variable from $d$ to 0 , during the armature movement, the quantity to be displayed is $y=d-x$, the moving armature displacement/position. That's why, it is necessary to capture the signal from the bond graph section of the model, by help of the $q$ sensor (generalized displacement sensor) and introduced into a block diagram section. The displayed result of simulation is shown in figure 13 , with a zoom for the small values of time.
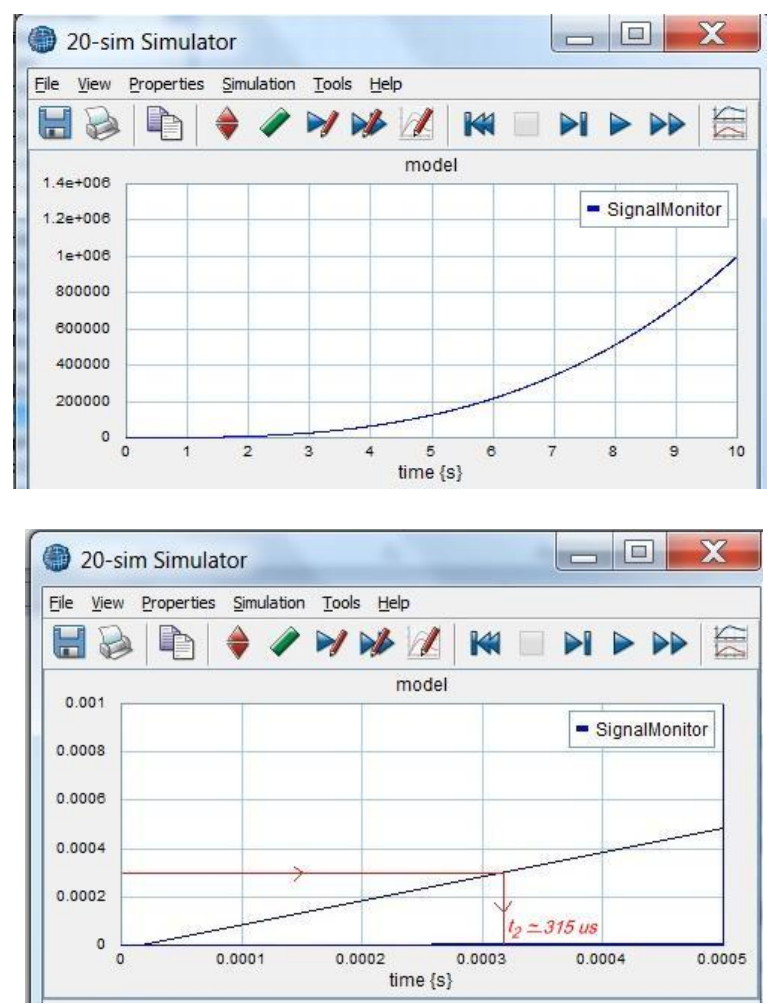

Fig. 13. Simulated displacement of the actuator

It is easy to notice that the result is closed enough to the ones obtained in [5], as it is presented in figure 14.

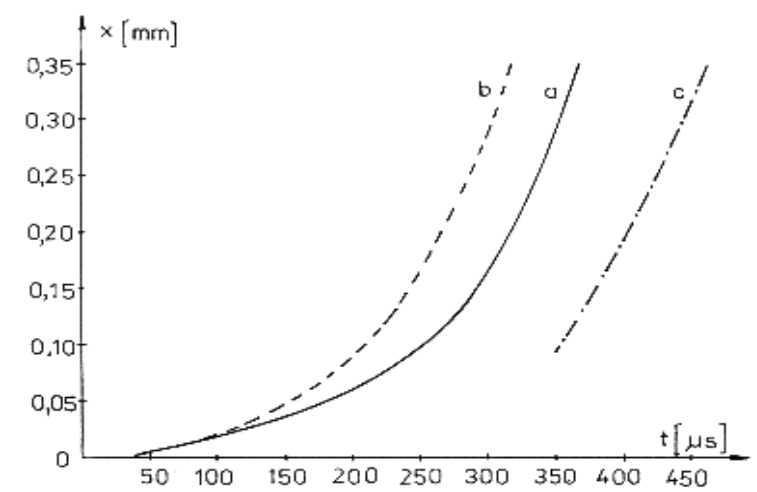

Fig. 14. Calculated and experimental results: $a-$ calculated with new method; $b$-calculated with Ter-Akopov method; c-experimental

\section{Conclusion}

Even there is an obvious need for analytical relationships to characterize the dynamic behaviour of the electromagnetic actuators, which could serve for preliminary design and optimization, as well as for use in control algorithms, numerical models are essential for an accurate evaluation of any physical system dynamics.

The bond graphs model, proposed in this paper, is an easy to develop one for any geometrical configuration of an electromagnetic actuator, while it conserves the physical structure of the system, for better understanding of the physical phenomena which rule the system behavior. The experiments confirmed the good results when using this model, compared to a well known analytical method, as well as a new developed one. The friendly approach within the 20sim software environment, which also allows user-defined submodels for facilitating the programming of the diverse needs for physical systems modelling is an undisputable advantage. Moreover, an easy to use graphical programming, having the possibilty to combine power models with signal blocks, is an easy way to get the resukts, without writing down equationds.

\section{References}

[1] Dong-Kyu, S. and colab.: Analysis of an Electromagnetic Actuator for Circuit Breakers. Journal of Electrical Engineering \& Technology. 2,3, pp. 346-352, (2007).

[2] Petit, L., and colab.: Design and Characterization of a High-Precision Digital Electromagnetic Actuator with Four Discrete Positions. Actuators. 4, pp.217-236; (2015).

[3] Niţu, Ctin., Niţu S. and Grămescu, B.: Electromagnetic actuator modeling for modular robotics applications. Journal of Optoelectronics and Advanced Materials, 10, 5, pp. 1137-1142, (2008).

[4] Stewart, P., Gladwin, D., and Fleming, P. J.: Multiobjective analysis for the design and control of an electromagnetic valve actuator. Proc. IMechE Vol. 221 Part D, pp.567-577.

[5] Niţu, Ctin. and Smaranda Niţu, An Improved Analytical Model for Electromagnetic Actuators Design. Elektronika, 8-9, pp.47-49, ISSN 00332089; (2004).

[6] Karnopp, D.C., Margolis, D.L. and Rosenberg, R.C. System Dynamics - Modeling and Simulation of Mechatronic Systems, John Wiley \& Sons Inc., New York, ISBN 0-471-33301-8; (2000).

[7] Schweer, J.: Berechnung kleiner WechselstromVentilmagnete mit massivem Eisenkreis, Dissertation, Universität Hannover, (1997). 